\title{
Quantum Noise and Superluminal Propagation
}

\author{
Bilha Segev \\ Department of Chemistry \\ Ben-Gurion University of the Negev \\ P.O.B. 653, Beer-Sheva 84105, Israel \\ Peter W. Milonni \\ Theoretical Division (T-4) \\ Los Alamos National Laboratory \\ Los Alamos, New Mexico 87545 \\ James F. Babb \\ Institute for Theoretical Atomic and Molecular Physics \\ Harvard-Smithsonian Center for Astrophysics \\ 60 Garden St. \\ Cambridge, Massachusetts 02138 \\ and \\ Raymond Y. Chiao \\ Department of Physics \\ University of California, Berkeley \\ Berkeley, California 94720
}




\begin{abstract}
Causal "superluminal" effects have recently been observed and discussed in various contexts. The question arises whether such effects could be observed with extremely weak pulses, and what would prevent the observation of an "optical tachyon." Aharonov, Reznik, and Stern (ARS) [Phys. Rev. Lett. 81, 2190 (1998)] have argued that quantum noise will preclude the observation of a superluminal group velocity when the pulse consists of one or a few photons. In this paper we reconsider this question both in a general framework and in the specific example, suggested by Chiao, Kozhekin, and Kurizki [Phys. Rev. Lett. 77, 1254 (1996)], of off-resonant, short-pulse propagation in an optical amplifier. We derive in the case of the amplifier a signal-to-noise ratio that is consistent with the general ARS conclusions when we impose their criteria for distinguishing between superluminal propagation and propagation at the speed $c$. However, results consistent with the semiclassical arguments of CKK are obtained if weaker criteria are imposed, in which case the signal can exceed the noise without being "exponentially large." We show that the quantum fluctuations of the field considered by ARS are closely related to superfluorescence noise. More generally we consider the implications of unitarity for superluminal propagation and quantum noise and study, in addition to the complete and truncated wavepackets considered by ARS, the residual wavepacket formed by their difference. This leads to the conclusion that the noise is mostly luminal and delayed with respect to the superluminal signal. In the limit of a very weak incident signal pulse, the superluminal signal will be dominated by the noise part, and the signal-to-noise ratio will therefore be very small.
\end{abstract}




\section{Introduction}

Chiao et al. [1], [2], [3] have shown that certain "superluminal" effects are possible without violation of standard notions of Einstein causality, i.e., without conveying information faster than the velocity $c$ of light in vacuum. Such effects have been demonstrated experimentally in optical tunneling [4], [5], [6] and in an electric circuit [7].

It has been suggested by Chiao, Kozhekin, and Kurizki (CKK) [1] that an optical pulse can propagate superluminally in an amplifier whose relaxation times are long compared with the pulse duration. The dispersion relation they derive can be obtained directly, as follows, starting from the formula for the refractive index of a monatomic gas:

$$
n(\omega)=1+\frac{2 \pi e^{2}}{m} \sum_{i} \sum_{j} \frac{N_{i} f(i, j)}{\omega_{j i}^{2}-\omega^{2}}
$$

for $n(\omega) \cong 1$, where $N_{i}$ is the number density of atoms in state $i$ and $f(i, j)$ is the oscillator strength for absorption on the $i \rightarrow j$ transition of frequency $\omega_{j i}$. Near a two-level resonance this becomes

$$
n(\omega)=1+\frac{2 \pi e^{2} f}{m} \frac{N_{1}-N_{2}}{\omega_{o}^{2}-\omega^{2}}
$$

where 1 and 2 designate the lower- and upper-energy levels, respectively, and $\omega_{o}=\omega_{21}$. Close to the transition resonance frequency $\omega_{o}$,

$$
n(\omega) \cong 1+\frac{\pi e^{2} f}{m \omega_{o}} \frac{N_{1}-N_{2}}{\omega_{o}-\omega-i \beta}
$$

when we include a dipole damping rate $\beta$. The (real) refractive index near a resonance is then

$$
n_{R}(\omega)=1+\frac{\pi e^{2} f}{m \omega_{o}} \frac{\omega_{o}-\omega}{\left(\omega_{o}-\omega\right)^{2}+\beta^{2}}\left(N_{1}-N_{2}\right) .
$$

Introducing the inversion $w=\left(N_{2}-N_{1}\right) / N$, where $N$ is the number density of atoms, and assuming a field sufficiently far from resonance that $\left(\omega_{o}-\omega\right)^{2}>>\beta^{2}$, we have

$$
\begin{gathered}
n_{R}(\omega) \cong 1-\frac{\pi e^{2} N w f}{m \omega_{o}} \frac{1}{\omega_{o}-\omega}, \\
k=n_{R}(\omega) \frac{\omega}{c}=\frac{\omega}{c}\left[1-\frac{\pi e^{2} N w f}{m \omega_{o}} \frac{1}{\omega_{o}-\omega}\right]=\frac{\omega}{c}\left[1-\frac{\omega_{p}^{2} w / 4 \omega_{o}}{\omega_{o}-\omega}\right], \\
k-k_{o}=\frac{1}{c}\left(\omega-\omega_{o}\right)-\frac{\omega}{c} \frac{\omega_{p}^{2} w / 4 \omega_{o}}{\omega_{o}-\omega} \cong \frac{1}{c}\left(\omega-\omega_{o}\right)-\frac{\omega_{p}^{2} w / 4 c}{\omega_{o}-\omega},
\end{gathered}
$$

and

$$
\Omega^{2}-K c \Omega+\frac{1}{4} w \omega_{p}^{2}=0,
$$


where $K=k-k_{o}, \Omega=\omega-\omega_{o}$, and the "plasma frequency" $\omega_{p}$ is defined by

$$
\omega_{p}^{2}=4 \pi N e^{2} f / m=8 \pi N d^{2} \omega_{o} / \hbar
$$

with $d$ the electric dipole transition moment. Equation (8) is the dispersion relation obtained by CKK.

We refer the reader to the CKK paper for a discussion of this dispersion relation. Here we simply note that (7) implies the group velocity

$$
v_{g}=\frac{d \omega}{d k}=c\left[1-\frac{\omega_{p}^{2} w / 4}{\left(\omega_{o}-\omega\right)^{2}}\right]^{-1}
$$

so that, in the case of an amplifier $(w>0)$, a short off-resonant pulse can propagate with a group velocity $v_{g}>c$.

Questions have been raised about the validity of the latter prediction at the one-photon level, which would correspond to what CKK call an "optical tachyon" [1. Aharonov, Reznik, and Stern (ARS) [8] have presented general arguments, based on the unitary evolution of the state vector, that "strongly questions the possibility that these systems may have tachyonlike quasiparticle excitations made up of a small number of photons." They also consider a particular model as an analog of the CKK system.

In this paper we address the question of superluminal propagation at the oneor few-photon level, and in particular the role played by quantum noise in the propagation of such extremely weak pulses. We begin in the following section with some physical considerations about the observability of superluminal propagation, and we briefly compare the ARS and CKK models. In Section 3 we formulate the Heisenberg equations of motion for the propagation of a short optical pulse in an inverted medium, and briefly review some relevant results from the theory of superfluorescence ( $\mathrm{SF}$ ). In Section 4 we derive a signal-to-noise ratio for the case where an incident, Gaussian signal pulse made up of $q$ photons is very short compared with the radiative lifetime and has a central frequency far removed from the resonance frequency of the medium. If we impose the ARS criterion for distinguishing between superluminal propagation and propagation at the speed of light, we find, consistent with their conclusions, that the signal must be "exponentially large" in order to distinguish it from quantum noise. If the ARS criterion is replaced by a much weaker one, however, the signal-to-noise ratio can exceed unity even for a one-photon signal pulse, as suggested by CKK. We relate the amplified quantum field fluctuations of ARS to quantum fluctuations of the atomic dipoles in the case of the optical amplifier. In Section 5, following the ideas of ARS, we present some general considerations based on the premises of unitarity and superluminal propagation. ARS show that, when the group velocity exceeds the speed of light, the superluminal signal is reconstructed from a truncated initial wavepacket, and that this truncated wavepacket has unstable modes. We show that the truncated wavepacket introduced by ARS propagates with both luminal and superluminal parts, and that, while the superluminal part is the reconstructed signal, the luminal part has the exponentially growing parts corresponding to the unstable modes. In addition, we study the residual wavepacket formed by the 
difference of the complete and truncated wavepackets. We show that contributions from the truncated and residual wavepackets cancel in the luminal region, but that, unlike the signal, the noise does not cancel, leading to the conclusion that the quantum noise is mostly luminal rather than superluminal. In the limit of a very weak incident signal pulse the signal-to-noise ratio will be very small, consistent with the conclusions reached by ARS.

It may be worth recalling that a primary reason for rejecting the possibility of superluminal transmission of information is the requirement that causality be maintained when Lorentz transformations are made: superluminal transmission of information would allow an event A causing an event B in one reference frame to occur after event B in a different frame. Considerations of superluminal propagation therefore often raise questions relating to Lorentz invariance. When and how should one include relativistic effects in order to ensure that physically meaningful results are obtained?

As in all previous treatments of pulse propagation in an inverted medium that we know of, we choose the reference frame in which the atoms are at rest. The Lorentz invariance of the fundamental, fully relativistic theory implies, of course, that our conclusions do not depend on this specific choice of a reference frame. Working in this frame, we treat the response of the atoms to the field in the approximation of nonrelativistic quantum mechanics. The electromagnetic field in this frame is also treated approximately, namely in the slowly varying envelope approximation that is used practically universally in the theory of resonant atom-field interactions. A different choice of reference frame would require us to start with the fully Lorentz-invariant equations and then make the slowly-varying-envelope and other approximations as appropriate. These approximations are known to be very accurate unless, for instance, the light pulse is extremely short, and to the extent that they are valid our results and conclusions are Lorentz-invariant.

\section{Preliminary Considerations}

The quantum noise limitations to superluminal propagation discussed by ARS were associated physically with spontaneous emission in the case of an optical amplifier, and could invalidate the CKK results in two ways. First, CKK assume that the atoms stay in their excited states as the pulse propagates through the amplifier. Radiative decay of the excited state will modify their "tachyonic dispersion relation" and, if the decay is rapid enough, can lead to a subluminal rather than superluminal group velocity, since $w$ in equation (10) can become negative. This can be avoided by using a sufficiently short pulse. Second, spontaneously emitted radiation might interfere with the measurement of the superluminal group velocity by introducing substantial noise. It is this possibility that is addressed by ARS.

Although the ARS arguments are certainly compelling, they are based in part on an analog of an optical amplifier rather than a theory involving the interaction of the electromagnetic field with an atomic medium. In particular, theirs is a model of a single quantum field rather than coupled atomic and electromagnetic quantum fields. The dispersion relation associated with this model, and the criteria assumed by ARS for the observability of superluminal propagation, lead to the conclusion, 
by analogy to an optical amplifier, that spontaneous emission noise cannot be avoided no matter how short the pulse or the transit time through the amplifier. Specifically, the unstable modes appearing in their model - which "are analogous to spontaneous emission in the optical model of an inverted medium of two-level systems" [8 - will preclude the observation of superluminal group velocity when the pulse is made up of a small number of photons; the quantum noise will be larger than the signal. In this section we present some physical considerations, motivated by the CKK and ARS analyses, for the observability of superluminal group velocity.

Following their equation (11), ARS state two necessary conditions for the observability of superluminal propagation $[c=1$ in their units]:

(1) $v_{g} T>>1 / \delta k$, where $v_{g}$ is the group velocity, $T$ is the time at which the wavepacket is observed, and $\delta k$ is the spectral width of their initial pulse.

(2) $\left(v_{g}-1\right) T>>1 / \delta k$

The first condition ensures that "the point of observation [is] far outside the initial spread of the wavepacket." The second allows us to "distinguish between superluminal propagation and propagation at the speed of light."

In the ARS model, where the field $\phi$ satisfies

$$
\frac{\partial^{2} \phi}{\partial t^{2}}-\frac{\partial^{2} \phi}{\partial z^{2}}-m^{2} \phi=0
$$

the group velocity is

$$
v_{g}=\frac{k_{o}}{\sqrt{k_{o}^{2}-m^{2}}},
$$

where $k_{o}$ is the central value of the spatial frequency $k$ for the initial pulse. For $m<k_{o}$ we can approximate $v_{g}$ by $1+m^{2} / 2 k_{o}^{2}$, so that condition 2 [and also condition 1] is satisfied if

$$
m^{2} T>>k_{o}^{2} / \delta k>>k_{o}
$$

$k_{o}>>1 / T$ - the condition that the observation time should be much larger than the central frequency of the pulse - then implies

$$
m T>>1
$$

Since for $m T>>1$ the amplified quantum noise grows exponentially [see Section 3], ARS conclude that the "signal amplitude should be exponentially large" in order to distinguish it from noise. Thus, according to ARS, the observability of superluminality for an input pulse consisting of only a few photons would be clouded by spontaneous emission noise.

Consider now the implications of conditions 1 and 2 for the actual system of interest, namely a very short optical pulse in an inverted medium. Can we satisfy these conditions for observation times short compared with the radiative lifetime? 
For a short optical pulse of central frequency $\omega$ propagating in an inverted medium $(w=1)$ with resonance frequency $\omega_{o}$, the refractive index is [equation (6)]

$$
n(\omega) \cong 1+\frac{2 \pi N d^{2} / \hbar}{\omega-\omega_{o}} \equiv 1-\frac{\omega_{p}^{2}}{4 \omega_{o} \Delta}
$$

for $\omega_{p}^{2} /\left(4 \omega_{o}\right)<<\left|\omega_{o}-\omega\right| \equiv|\Delta|$. We are assuming that $|\Delta|$ is large compared with the absorption width, which in our case is the radiative decay rate. Equation (15) implies

$$
\frac{v_{g}}{c}=\left(\frac{d}{d \omega}[\omega n(\omega)]\right)^{-1}=\frac{1}{1-\omega_{p}^{2} / 4 \Delta^{2}}
$$

and

$$
\frac{v_{g}}{c}-1=\frac{\omega_{p}^{2} / 4 \Delta^{2}}{1-\omega_{p}^{2} / 4 \Delta^{2}}=\frac{\omega_{p}^{2}}{4 \Delta^{2}} \frac{v_{g}}{c} .
$$

Then conditions 1 and 2 of ARS become, respectively,

$$
\begin{aligned}
& \frac{T}{1-\omega_{p}^{2} / 4 \Delta^{2}}>>\frac{1}{c \delta k} \sim \tau_{p}, \\
& \frac{\left(\omega_{p}^{2} / 4 \Delta^{2}\right) T}{1-\omega_{p}^{2} / 4 \Delta^{2}}>>\frac{1}{c \delta k} \sim \tau_{p},
\end{aligned}
$$

with $\tau_{p}$ the pulse duration. Both conditions can be satisfied if, for instance, $T>>\tau_{p}$ and $\omega_{p}^{2} / 4 \Delta^{2}$ is not too small. To avoid spontaneous emission during the observation time $T$, take $T<<\tau_{R A D}$, where $\tau_{R A D}$ is the radiative lifetime of a single inverted atom. Then the ARS conditions require that

$$
\tau_{R A D}>T>>\tau_{p}
$$

As noted by CKK, there is another aspect of an inverted atomic medium that must be addressed, namely superfluorescence (SF). SF is a collective phenomenon of the sample as a whole. We shall denote by $N_{T}, S$, and $L$ the number of atoms, the cross-sectional area, and the length of the sample, respectively, so that the density of atoms is given by $N=N_{T} / S L$. If collisional and other dephasing mechanisms are sufficiently weak, an inverted medium of $N_{T}$ atoms can emit SF radiation at the rate

$$
\tau_{R}=\tau_{R A D} / N_{T}
$$

i.e., the radiative decay time can in effect be smaller by a factor of $N_{T}$ than the single-atom radiative lifetime $\tau_{R A D}$ assumed in the discussion thus far. The peak of the SF pulse occurs at a time [9]

$$
\tau_{D} \sim \tau_{R}\left[\frac{1}{4} \ln \left(2 \pi N_{T}\right)\right]^{2}
$$

following the excitation of the atoms. It would appear then that the quantum noise associated with SF will be small if

$$
\tau_{p}, L / c<\tau_{R}<\tau_{D}
$$


We note for later purposes that

$$
\omega_{p}^{2}=\frac{8 \pi N d^{2} \omega_{o}}{\hbar}=\frac{1}{\tau_{R A D}} \frac{N_{T}}{S L} S c=\frac{4}{\tau_{R}} \frac{c}{L},
$$

where we have used equation (131) of Appendix A for the single-atom radiative lifetime $\tau_{R A D}$.

This brief summary lends support to the CKK suggestion, but obviously a more quantitative analysis is called for. To this end we now formulate, in the Heisenberg picture, the quantum theory of pulse propagation in an amplifier.

\section{Formalism for Pulse Propagation}

We begin with the Hamiltonian for $N_{T}$ two-level atoms (TLAs) interacting with the quantized electromagnetic field via electric dipole transitions:

$$
\hat{H}=\frac{1}{2} \hbar \omega_{o} \sum_{j=1}^{N_{T}} \hat{\sigma}_{z j}-d \sum_{j=1}^{N_{T}} \hat{\sigma}_{x j} \hat{E}\left(z_{j}\right)+\sum_{k} \hbar \omega_{k} \hat{a}_{k}^{\dagger} \hat{a}_{k}
$$

where $\omega_{o}$ and $d$ have the same meaning as before and $z_{j}$ is the $z$-coordinate of atom $j$. The carets $\left(^{\wedge}\right)$ are used to denote operators. We consider a one-dimensional model in which the atoms occupy the region from $z=0$ to $z=L$ and the field is a superposition of plane waves propagating in the $z$ direction. The electric field operator is given by $\hat{E}(z)=\hat{E}^{(+)}(z)+\hat{E}^{(-)}(z)$, where

$$
\hat{E}^{(+)}(z)=i \sum_{k}\left(\frac{2 \pi \hbar \omega_{k}}{S \ell}\right)^{1 / 2} \hat{a}_{k} e^{i k z} \quad\left(k=\omega_{k} / c\right)
$$

and $\hat{E}^{(-)}(z)=\hat{E}^{(+)}(z)^{\dagger}$. S $S$, where $S$, as before, is a cross-sectional area and $\ell$ a length, is the quantization volume. For simplicity we consider only a single field polarization, namely linear polarization along the direction of the transition dipole moment of the TLAs. $\hat{a}_{k}$ and $\hat{a}_{k}^{\dagger}$ are the photon annihilation and creation operators, respectively, for mode $k$, and the $\hat{\sigma}^{\prime}$ 's are the Pauli two-state operators in the standard notation.

We will work in the Heisenberg picture, in which the time-dependent electric field operator satisfies

$$
\left(\frac{\partial^{2}}{\partial z^{2}}-\frac{1}{c^{2}} \frac{\partial^{2}}{\partial t^{2}}\right) \hat{E}=\frac{4 \pi}{c^{2}} \frac{\partial^{2} \hat{P}}{\partial t^{2}}=\frac{4 \pi d}{c^{2} S} \sum_{j=1}^{N_{T}} \frac{\partial^{2} \hat{\sigma}_{x j}}{\partial t^{2}} \delta\left(z-z_{j}\right) \rightarrow \frac{4 \pi}{c^{2}} N d \frac{\partial^{2}}{\partial t^{2}} \hat{\sigma}_{x}(z, t)
$$

where in the last step we have made the continuum approximation for the polarization density $\hat{P}$, assuming a uniform atomic density $N$. We now write

$$
\hat{E}^{(+)}(z, t)=\hat{F}(z, t) e^{-i \omega(t-z / c)}
$$


and assume $\hat{F}(z, t)$ is slowly varying in $z$ and $t$ compared with $\exp [-i \omega(t-z / c)]$. In this approximation

$$
2 i \frac{\omega}{c}\left(\frac{\partial \hat{F}}{\partial z}+\frac{1}{c} \frac{\partial \hat{F}}{\partial t}\right)+h . c .=\frac{4 \pi}{c^{2}} N d \frac{\partial^{2} \hat{\sigma}_{x}}{\partial t^{2}} e^{i \omega(t-z / c)}
$$

It will be convenient to use the atomic lowering and raising operators $\hat{\sigma}=\frac{1}{2}\left(\hat{\sigma}_{x}-\right.$ $\left.i \hat{\sigma}_{y}\right)$ and $\hat{\sigma}^{\dagger}=\frac{1}{2}\left(\hat{\sigma}_{x}+i \hat{\sigma}_{y}\right)$, respectively, such that $\left[\hat{\sigma}, \hat{\sigma}^{\dagger}\right]=-\hat{\sigma}_{z}$, and to write

$$
\hat{\sigma}(z, t)=\hat{s}(z, t) e^{-i \omega(t-z / c)}
$$

where the operator $\hat{s}(z, t)$ is assumed to be slowly varying in the same sense as $\hat{F}(z, t)$. Then, in the rotating-wave approximation (RWA), we can replace (29) with

$$
\frac{\partial \hat{F}}{\partial z}+\frac{1}{c} \frac{\partial \hat{F}}{\partial t}=\left(2 \pi i N d \frac{\omega_{o}}{c}\right) \hat{s}
$$

where on the right-hand side we have approximated $\omega$ by $\omega_{o}$. This equation and the TLA Heisenberg equations

$$
\begin{gathered}
\frac{\partial \hat{s}}{\partial t}=-i(\Delta-i \beta) \hat{s}-\frac{i d}{\hbar} \hat{\sigma}_{z} \hat{F} \\
\frac{\partial \hat{\sigma}_{z}}{\partial t}=-2 \beta\left(1+\hat{\sigma}_{z}\right)-\frac{2 i d}{\hbar}\left(\hat{F}^{\dagger} \hat{s}-\hat{s}^{\dagger} \hat{F}\right)
\end{gathered}
$$

derived in Appendix A form a closed set of operator equations. They provide the basis for a quantum theory of propagation in either amplifying or absorbing media.

In the semiclasical approximation in which the atom and field operators are replaced by their expectation values, equations (31)-(33) reduce to well known Maxwell-Bloch equations. Otherwise, different limits can apply:

- The limit of $\beta \rightarrow 0, \Delta=0$ and $\hat{\sigma}_{z} \rightarrow 1$ considered below gives equations (35)-(37) implying superfluorescence when the initial state of the field is the vacuum.

- The limit of $\omega \gg \omega_{0}$ gives the ARS field equation, as discussed below.

- Finally, in Section 4 the CKK case of large detuning, $\hat{\sigma}_{z} \rightarrow 1$, and the initial state of a very short incoming pulse, is studied.

If the field central frequency $\omega$ is assumed to match exactly the atomic resonance frequency $\omega_{o}$, so that $\Delta=0$, and if we restrict ourselves to times short compared with the single-atom radiative lifetime $\left[\tau_{R A D}=(2 \beta)^{-1}\right]$ and assume that the atoms remain with probability $\cong 1$ in their excited states over times of interest, we can ignore (33) and replace $\hat{\sigma}_{z}(z, t)$ by 1 and equation (32) by

$$
\frac{\partial \hat{s}}{\partial t}=-\frac{i d}{\hbar} \hat{F}
$$


In terms of the independent variables $\zeta=t-z / c$ and $\eta=z$,

$$
\begin{gathered}
\frac{\partial \hat{s}}{\partial \zeta}=-\frac{i d}{\hbar} \hat{F} \\
\frac{\partial \hat{F}}{\partial \eta}=\left(2 \pi i N d \frac{\omega_{o}}{c}\right) \hat{s},
\end{gathered}
$$

implying

$$
\frac{\partial^{2} \hat{s}}{\partial \eta \partial \zeta}=\left(\frac{\omega_{p}^{2}}{4 c}\right) \hat{s}, \quad \frac{\partial^{2} \hat{F}}{\partial \eta \partial \zeta}=\left(\frac{\omega_{p}^{2}}{4 c}\right) \hat{F} .
$$

Equations (35)-(37) have been used in studies of the buildup of superfluorescent radiation [9]. It will be useful for the discussion in Section 4 to briefly rederive here one of the most important results of those studies.

Equation (31) has the formal solution

$$
\begin{aligned}
\hat{F}(z, t) & =\hat{F}_{o}(z, t)+\left(2 \pi i N d \frac{\omega_{o}}{c}\right) \int_{0}^{z} d z^{\prime} \hat{s}\left(z^{\prime}, t-\frac{z-z^{\prime}}{c}\right) \theta\left(t-\frac{z-z^{\prime}}{c}\right) \\
& =\hat{F}_{o}(z, t)+\left(2 \pi i N d \frac{\omega_{o}}{c}\right) \int_{0}^{z} d z^{\prime} \hat{s}\left(z-z^{\prime}, t-z^{\prime} / c\right) \theta\left(t-z^{\prime} / c\right)
\end{aligned}
$$

where we have chosen the retarded Green function over the advanced Green function in order to ensure causality. Here $\theta$ is the unit step function and $\hat{F}_{o}(z, t)$ is a solution of the homogeneous equation. We are interested here in the expectation value $\left\langle\hat{F}^{\dagger}(L, t) \hat{F}(L, t)\right\rangle$ at the end $(z=L)$ of the medium. For SF the expectation value is taken over the vacuum state of the field, in which case the first term on the right-hand side of (38) does not contribute to normally ordered expectation values. We may therefore ignore this term for practical purposes. Defining $y=2 \sqrt{\zeta \eta}$ we find from (37) that $\hat{s}$ satisfies the differential equation for $I_{0}(y)$, the modified Bessel function of order zero [10]. The solution of interest for $\hat{F}(L, t)$ is then 9

$$
\hat{F}(L, t)=\left(2 \pi i N d \frac{\omega_{0}}{c}\right) \int_{0}^{L} d z^{\prime} \hat{s}\left(L-z^{\prime}, 0\right) I_{0}\left(\omega_{p} \sqrt{\left(z^{\prime} / c\right)\left(t-z^{\prime} / c\right)}\right) \theta\left(t-z^{\prime} / c\right)
$$

In order to calculate $\left\langle\hat{F}^{\dagger}(L, t) \hat{F}(L, t)\right\rangle$ we require $\left\langle\hat{s}^{\dagger}\left(z^{\prime}, 0\right) \hat{s}(z, 0)\right\rangle$, which we evaluate in Appendix B. We obtain [9]

$$
\left\langle\hat{F}^{\dagger}(L, t) \hat{F}(L, t)\right\rangle=\left(2 \pi d \frac{\omega_{o}}{c}\right)^{2} \frac{N}{S} \int_{0}^{L} d x \theta(t-x / c) I_{0}^{2}\left(\omega_{p} \sqrt{(x / c)(t-x / c)}\right) .
$$

For times large enough that $I_{0}$ may be replaced by its asymptotic form,

$$
\left\langle\hat{F}^{\dagger}(L, t) \hat{F}(L, t)\right\rangle \sim \frac{1}{8 \pi} \frac{2 \pi \hbar \omega_{o}}{S c t} e^{\sqrt{t / \tau_{R}}}
$$

Equating the intensity expectation value $(c / 2 \pi)\left\langle\hat{F}^{\dagger}(L, t) \hat{F}(L, t)\right\rangle$ to the maximum expected SF intensity $N_{T} \hbar \omega_{o} / S \tau_{R}$, we arrive at the expression (22) for the time 
at which the SF pulse reaches its peak intensity. In the short-time limit, on the other hand,

$$
\left\langle\hat{F}^{\dagger}(L, t) \hat{F}(L, t)\right\rangle \sim\left(2 \pi d \frac{\omega_{o}}{c}\right)^{2} \frac{N}{S} c t,
$$

a result we will return to in Section 4.

\section{Approximation Leading to ARS Field Equation}

Our considerations thus far assume that the field central frequency lies in the vicinity of the atomic resonance in the sense that the detuning $\Delta$ is small in magnitude compared with $\omega$ and $\omega_{o}$. Let us now suppose instead that the field frequency $\omega$ is very large compared with $\omega_{o}$. In this case we must work with the atomic operators $\hat{\sigma}_{x}, \hat{\sigma}_{y}$ instead of the slowly varying $\hat{s}$. ¿From equations (120) and (121) of Appendix A we have

$$
\ddot{\hat{\sigma}}_{x}+\omega_{o}^{2} \hat{\sigma}_{x}=-\frac{2 d \omega_{o}}{\hbar} \hat{\sigma}_{z} \hat{E} \cong-\frac{2 d \omega_{o}}{\hbar} \hat{E}
$$

in the approximation $\hat{\sigma}_{z} \cong 1$. The assumption $\omega>>\omega_{o}$ implies

$$
\ddot{\hat{\sigma}}_{x} \cong-\frac{2 d \omega_{o}}{\hbar} \hat{E}
$$

so that, from equation (27),

$$
\left(\frac{\partial^{2}}{\partial t^{2}}-c^{2} \frac{\partial^{2}}{\partial z^{2}}-\omega_{p}^{2}\right) \hat{E}=0
$$

This is identical to the equation of motion for the quantum field in the ARS model when we equate $\omega_{p}^{2}$ to their $m^{2}$. From this perspective the ARS equation of motion describes the interaction of the electromagnetic field with $N$ unbound electrons $\left(\omega>>\omega_{0}\right)$ per unit volume. However, the usual plasma dispersion formula $n^{2}=$ $1-\omega_{p}^{2} / \omega^{2}$ for the refractive index $n$ is replaced in this case by

$$
n^{2}=1+\omega_{p}^{2} / \omega^{2} \text {. }
$$

This is a consequence of the assumption $\hat{\sigma}_{z} \cong 1$; had we assumed $\hat{\sigma}_{z} \cong-1$ we would have obtained the familiar plasma dispersion formula.

To describe the growth of the quantum noise with time in this model, we write (45) in the form

$$
\frac{\partial^{2} \hat{E}}{\partial \tau_{1} \partial \tau_{2}}-\frac{m^{2}}{4} \hat{E}=0,
$$

where $\tau_{1}=t-z / c, \tau_{2}=t+z / c$. In terms of the independent variable $y=m \sqrt{\tau_{1} \tau_{2}}$, equation(47) has solutions that are linear combinations of the zero-order modified Bessel functions $I_{0}(y), K_{0}(y)$. For large $t$, the vacuum expectation value

$$
\left\langle\hat{E}^{2}(z, t)\right\rangle \propto I_{0}^{2}(y) \sim \frac{e^{2 m t}}{2 \pi m t}
$$

so that the quantum noise grows exponentially in time from the initial fluctuations of the vacuum field, the fluctuations present before the medium in the ARS model is "inverted." 


\section{$4 \quad$ Signal and Noise}

We wish to determine to what extent the observation of the superluminal group velocity considered by CKK will be affected by quantum noise. The system of interest is described by the Heisenberg equations of motion (31) and (32). We approximate $\hat{\sigma}_{z}$ by 1 , assuming that pulse durations $\tau_{p}$ and transit times $L / c$ are sufficiently small that de-excitation of the initially inverted atoms by radiation (or any other decay process) is negligible. The situation here is different from that describing the onset of SF in that (a) the detuning $\Delta$ is not zero but is instead large (Section 2), and (b) the initial state of the field is not the vacuum but corresponds to a short pulse of radiation from some external source.

The equation for $\hat{s}(z, t)$ in the present model is

$$
\frac{\partial \hat{s}}{\partial t}=-i(\Delta-i \beta) \hat{s}-\frac{i d}{\hbar} \hat{F}
$$

or

$$
\hat{s}(z, t)=\hat{s}\left(z, t_{o}\right) e^{-i(\Delta-i \beta)\left(t-t_{o}\right)}-\frac{i d}{\hbar} \int_{t_{o}}^{t} d t^{\prime} \hat{F}\left(z, t^{\prime}\right) e^{i(\Delta-i \beta)\left(t^{\prime}-t\right)} .
$$

$t_{o}$ is some initial time, before any pulse is injected into the medium. We take $\hat{F}\left(z, t_{o}\right)=0$, although of course what this really means is that there is no nonvanishing field or intensity in the medium at $t_{o}$, so that for practical purposes (normally ordered expectation values) we can in effect ignore the operator $\hat{F}\left(z, t_{o}\right)$ in the equation for $\hat{s}(z, t)$.

The pulse is assumed to have a central frequency $\omega$ and to have no significant frequency components near the resonance frequency $\omega_{o}:|\Delta| \tau_{p}>1$. We assume that $|\Delta| \tau_{p}$ is large enough that we can approximate (50) by integrating by parts and retaining only the leading terms:

$$
\hat{s}(z, t) \cong \hat{s}\left(z, t_{o}\right) e^{-i(\Delta-i \beta)\left(t-t_{o}\right)}-\frac{d}{\hbar} \frac{\Delta+i \beta}{\Delta^{2}+\beta^{2}} \hat{F}(z, t)-\frac{i d}{\hbar \Delta^{2}} \frac{\partial \hat{F}}{\partial t} .
$$

As will be clear from the analysis that follows, this approximation implies the undistorted propagation of the incident pulse at the group velocity $v_{g}$, as assumed by CKK.

¿From (31),

$\frac{\partial \hat{F}}{\partial z}+\frac{1}{c} \frac{\partial \hat{F}}{\partial t} \cong\left(2 \pi i N d \frac{\omega_{o}}{c}\right) \hat{s}\left(z, t_{o}\right) e^{-i(\Delta-i \beta)\left(t-t_{o}\right)}+\frac{g}{2} \hat{F}+i[n(\omega)-1] \frac{\omega}{c} \hat{F}+\left(\frac{1}{c}-\frac{1}{v_{g}}\right) \frac{\partial \hat{F}}{\partial t}$,

where

$$
g \equiv \frac{4 \pi N d^{2} \omega_{o}}{\hbar c} \frac{\beta}{\Delta^{2}+\beta^{2}}
$$

is the gain coefficient for propagation of a field with frequency $\omega$ in the inverted medium. We have used equation (15) for the refractive index $n(\omega)$ and (17) for $v_{g} / c-1$. Writing $\hat{F}(z, t)=\hat{F}^{\prime}(z, t) e^{i[n(\omega)-1] \omega z / c}$ and $\hat{s}\left(z, t_{o}\right)=\hat{s}^{\prime}\left(z, t_{o}\right) e^{i[n(\omega)-1] \omega z / c}$ 
yields an equation in terms of the primed variables in which the term $i[n(\omega)-$ $1](\omega / c) z$ associated with phase velocity is eliminated. Then, ignoring for practical purposes the difference between the primed and unprimed variables, we have

$$
\frac{\partial \hat{F}}{\partial z}+\frac{1}{v_{g}} \frac{\partial \hat{F}}{\partial t}=\frac{g}{2} \hat{F}+\left(2 \pi i N d \frac{\omega_{o}}{c}\right) \hat{s}\left(z, t_{o}\right) e^{-i(\Delta-i \beta)\left(t-t_{o}\right)},
$$

and therefore

$$
\begin{aligned}
\hat{F}(z, t) & =\hat{F}\left(0, t-z / v_{g}\right) e^{g z / 2}+\left(2 \pi i N d \frac{\omega_{o}}{c}\right) \int_{0}^{z} d z^{\prime} \hat{s}\left(z^{\prime}, t_{o}\right) e^{g\left(z-z^{\prime}\right) / 2} \\
& \times e^{-i(\Delta-i \beta)\left[t-t_{o}-\left(z-z^{\prime}\right) / v_{g}\right]} \theta\left(t-t_{o}-\left(z-z^{\prime}\right) / v_{g}\right) \\
& \equiv \hat{F}_{s}\left(0, t-z / v_{g}\right) e^{g z / 2}+\hat{F}_{n}(z, t)
\end{aligned}
$$

where the subscripts $\mathrm{s}$ and $\mathrm{n}$ denoted signal and noise, respectively. Here

$\hat{F}_{n}(z, t)=\left(2 \pi i N d \frac{\omega_{o}}{c}\right) \int_{0}^{z} d z^{\prime} \hat{s}\left(z^{\prime}, t_{o}\right) e^{g\left(z-z^{\prime}\right) / 2} e^{-i(\Delta-i \beta)\left[t-t_{o}-\left(z-z^{\prime}\right) / v_{g}\right]} \theta\left(t-t_{o}-\left(z-z^{\prime}\right) / v_{g}\right)$

is a quantum noise field associated with the quantum fluctuations of the atomic dipoles.

To appreciate the significance of $g$ as defined by equation (53), consider the gain coefficient $g_{R}$ for a radiatively broadened transition of frequency $\omega_{o}$ and radiative decay rate $1 / \tau_{R A D}=2 \beta$. For light of frequency $\omega=\omega_{o}-\Delta$,

$$
g_{R}=\frac{N S}{\tau_{R A D}} \frac{2 \beta}{\Delta^{2}+\beta^{2}}=\frac{4 \pi N d^{2} \omega_{o}}{\hbar c} \frac{\beta}{\Delta^{2}+\beta^{2}}
$$

if we assume that all the $N$ atoms per unit volume are in the upper state of the amplifying transition. Thus $g_{R}=g$, i.e., $g$ is just the gain coefficient for amplification by stimulated emission. We note also that, from equation (17),

$$
g=2 \beta\left(\frac{1}{c}-\frac{1}{v_{g}}\right)
$$

in the case under consideration where the amplifying transition is radiatively broadened and the detuning is large compared with the gain bandwidth.

The operator $\hat{s}\left(z, t_{o}\right)$ has the expectation-value properties (140) and (141) of Appendix B. These properties imply $\left\langle\hat{F}_{n}(z, t)\right\rangle=\left\langle\hat{F}_{n}^{\dagger}(z, t)\right\rangle=0$ and

$$
\begin{aligned}
\left\langle\hat{F}_{n}^{\dagger}(z, t) \hat{F}_{n}(z, t)\right\rangle & =\left(2 \pi N d \frac{\omega_{o}}{c}\right)^{2} \frac{L}{N_{T}} e^{-2 \beta\left(t-t_{o}\right)} \int_{z-v_{g}\left(t-t_{o}\right)}^{z} d z^{\prime} e^{g\left(z-z^{\prime}\right)} e^{2 \beta\left(z-z^{\prime}\right) / v_{g}} \\
& =\left(2 \pi d \frac{\omega_{o}}{c}\right)^{2} \frac{N}{S} \frac{c}{2 \beta}\left[e^{g v_{g} t}-e^{-2 \beta t}\right]
\end{aligned}
$$

where we have used the relations (58) and $N_{T}=N S L$ and, to simplify the notation, we have taken $t_{o}=0$. 
Since the atom and field are initially uncorrelated, i.e.,

$$
\left\langle\hat{F}^{\dagger}\left(0, t-z / v_{g}\right) \hat{s}_{j}\left(t_{o}\right)\right\rangle=\left\langle\hat{F}_{n}^{\dagger}\left(0, t-z / v_{g}\right)\right\rangle\left\langle\hat{s}_{j}\left(t_{o}\right)\right\rangle=0
$$

we have, at the end of the amplifier,

$$
\left\langle\hat{F}^{\dagger}(L, t) \hat{F}(L, t)\right\rangle=\left\langle\hat{F}_{s}^{\dagger}\left(0, t-L / v_{g}\right) \hat{F}_{s}\left(0, t-L / v_{g}\right)\right\rangle e^{g L}+\left\langle\hat{F}_{n}^{\dagger}(L, t) \hat{F}_{n}(L, t)\right\rangle
$$

and the signal-to-noise ratio

$$
\begin{aligned}
S N R(L, t) & \equiv \frac{\left\langle\hat{F}_{s}^{\dagger}\left(0, t-L / v_{g}\right) \hat{F}_{s}\left(0, t-L / v_{g}\right)\right\rangle e^{g L}}{\left\langle\hat{F}_{n}^{\dagger}(L, t) \hat{F}_{n}(L, t)\right\rangle} \\
& =\frac{\left\langle\hat{F}_{s}^{\dagger}\left(0, t-L / v_{g}\right) \hat{F}_{s}\left(0, t-L / v_{g}\right)\right\rangle e^{g L}}{\left(2 \pi d \omega_{o} / c\right)^{2}(N / S)(c / 2 \beta)\left[e^{g L}-e^{\left.-2 \beta L / v_{g}\right]}\right.} \\
& =\frac{\left\langle\hat{F}_{s}^{\dagger}\left(0, t-L / v_{g}\right) \hat{F}_{s}\left(0, t-L / v_{g}\right)\right\rangle e^{2 \beta\left(1 / c-1 / v_{g}\right) L}}{\left(2 \pi d \omega_{o} / c\right)^{2}(N / S)(c / 2 \beta)\left[e^{2 \beta\left(1 / c-1 / v_{g}\right) L}-e^{-2 \beta L / v_{g}}\right]} \\
& \cong \frac{\left\langle\hat{F}_{s}^{\dagger}\left(0, t-L / v_{g}\right) \hat{F}_{s}\left(0, t-L / v_{g}\right)\right\rangle}{\left(2 \pi d \omega_{o} / c\right)^{2} N L / S} .
\end{aligned}
$$

In the denominators we have taken $t=L / v_{g}$ for the time over which the atoms radiate, and have used the fact that $2 \beta\left(1 / c-1 / v_{g}\right) L=g L$, the difference of two numbers that themselves are small according to our assumption that propagation times are small compared with the single-atom radiative decay rate, is $<<1$.

The numerator in equation (62) can be related to the expectation value $q$ of the number of photons in the incident signal pulse as follows. The expectation value of the incident signal intensity is

$$
I_{s}(0, t)=\frac{v_{g}}{2 \pi}\left\langle\hat{F}_{s}(0, t) \hat{F}_{s}(0, t)\right\rangle=I_{o} e^{-t^{2} / \tau_{p}^{2}}
$$

for a Gaussian pulse of duration $\tau_{p}$. Requiring that the energy flux $\int_{-\infty}^{\infty} d t I_{s}(z, t)$ be $q \hbar \omega / S \cong q \hbar \omega_{o} / S$ implies $I_{o}=q \hbar \omega_{o} /\left(S \tau_{p} \sqrt{\pi}\right)$ and therefore

$$
\left\langle\hat{F}_{s}^{\dagger}\left(0, t-L / v_{g}\right) \hat{F}_{s}\left(0, t-L / v_{g}\right)\right\rangle=q \frac{2 \pi \hbar \omega_{o}}{v_{g} S \tau_{p} \sqrt{\pi}} e^{-\left(t-L / v_{g}\right)^{2} / \tau_{p}^{2}}
$$

Thus

$$
\begin{aligned}
S N R(L, t) & =\frac{q}{\tau_{p} \sqrt{\pi}} \frac{c}{v_{g}} e^{-\left(t-L / v_{g}\right)^{2} / \tau_{p}^{2}}\left(\frac{2 \pi d^{2} \omega_{o}}{\hbar c S} N S L\right)^{-1} \\
& =\frac{q}{\sqrt{\pi}}\left(\frac{4 c}{\omega_{p}^{2} L \tau_{p}}\right) \frac{c}{v_{g}} e^{-\left(t-L / v_{g}\right)^{2} / \tau_{p}^{2}} \\
& =\frac{q}{\sqrt{\pi}} \frac{\tau_{R}}{\tau_{p}} \frac{c}{v_{g}} e^{-\left(t-L / v_{g}\right)^{2} / \tau_{p}^{2}}
\end{aligned}
$$


where we have used equation (24) [11.

Among the criteria given by $\mathrm{CK} \overline{\mathrm{K}}$ for the observation of a superluminal pulse is that "The probe-pulse duration $\left[\tau_{p}\right]$ must not exceed $\tau_{R}=4 c / L \omega_{p}^{2}$." This criterion implies, from equation (65), that $\operatorname{SNR}(L, t) \geq(q / \sqrt{\pi}) c / v_{g}$ and therefore that it is possible, even for $q \sim 1$, to have superluminal propagation with $S N R(L, t)>1$ if the pulse duration is short enough: $\tau_{p}<\tau_{R} c / v_{g}$.

In order to relate this conclusion to ARS, we use equation (17) to write (65) as

$$
S N R(L, t)=\frac{q}{\sqrt{\pi}} \frac{\tau_{p}}{\left(\frac{v_{g}}{c}-1\right) \frac{L}{c} \Delta^{2} \tau_{p}^{2}} e^{-\left(t-L / v_{g}\right)^{2} / \tau_{p}^{2}} .
$$

We see from this expression that, if we impose the ARS condition (2), i.e., $\left(v_{g} / c-\right.$ 1) $L / c>>\tau_{p}$, then

$$
\operatorname{SNR}(L, t)<<\frac{q}{\sqrt{\pi}} \frac{1}{\left(\Delta \tau_{p}\right)^{2}} e^{-\left(t-L / v_{g}\right)^{2} / \tau_{p}^{2}}
$$

so that, given also the condition on $|\Delta| \tau_{p}$ discussed before equation (51), the signalto-noise ratio will be very small when the ARS condition for strong distinguishability of superluminal propagation from propagation at the speed $c$ is satisfied. then

In fact if $\left(v_{g} / c-1\right) L / c>>\tau_{p}$ and therefore $\operatorname{SNR}(L, t)$ is very small for $q \approx 1$,

$$
t / \tau_{R}=\frac{L / c}{\tau_{R}}=\frac{v_{g}}{c} \frac{L / v_{g}}{\tau_{R}} \gtrsim \frac{v_{g}}{c} \frac{\tau_{p}}{\tau_{R}},
$$

which, from (65), must be large. Then the SF noise must be exponentially large [equation (41)]. It follows that $q$ must be exponentially large in order to maintain a signal-to-noise ratio greater than unity. This is consistent with the ARS conclusion that "for the signal amplitude to be larger than the amplitude of the fluctuations at the observation time, the signal amplitude should be exponentially large" [8].

Our results are therefore in agreement with ARS in that, if we require the separation of the superluminal pulse and a twin vacuum-propagated pulse to be much larger than the pulse duration, the signal-to-noise ratio will be very small at the one- or few-photon level. On the other hand, the results are not inconsistent with CKK: even at the one-photon level we can achieve a signal-to-noise ratio greater than unity if this separation $\left(\left[v_{g} / c-1\right] L / c\right)$ is smaller than the pulse duration $\tau_{p}$ [Equation (66)].

\section{Physical Origin of the Noise Limiting the Observation of Superluminal Group Velocity}

Note that, when we set the time $t$ in equation (42) for the short-time SF noise intensity equal to the "observation time" $L / c$, we obtain exactly the noise intensity appearing in the denominator of equation (62) [12]. Thus the quantum noise that imposes limitations on the observation of superluminal group velocity is attributable to the initiation of SF. 


\section{Operator Ordering and Relation to ARS Approach}

Less obvious, perhaps, is the relation between the quantum noise we have considered - which stems from the atomic dipole fluctuations characterized by equations (140) and (141) of Appendix B - and the quantum noise of ARS, which is attributed to the quantum fluctuations of the field.

To establish the relation to the ARS approach we return to our calculation of the noise intensity, using now anti-normally ordered field operators instead of the normally ordered operators used before. Thus we consider now the expectation value $\left\langle\hat{F}(z, t) \hat{F}^{\dagger}(z, t)\right\rangle$ instead of $\left\langle\hat{F}^{\dagger}(z, t) \hat{F}(z, t)\right\rangle$. In this approach the atomic dipole fluctuations play no explicit role, as can be seen from equation (55) and the fact that

$$
\left\langle\hat{s}\left(z^{\prime}, t_{o}\right) \hat{s}^{\dagger}\left(z^{\prime \prime}, t_{o}\right)\right\rangle=0
$$

for excited atoms. In this case, however, the initially unoccupied modes of the field make a nonvanishing contribution as a consequence of non-normal ordering:

$$
\begin{aligned}
\left\langle\hat{F}\left(0, t-L / v_{g}\right) \hat{F}^{\dagger}\left(0, t-L / v_{g}\right)\right\rangle & =\sum_{k} \frac{2 \pi \hbar \omega_{k}}{S \ell}\left\langle\hat{a}_{k}(0) \hat{a}_{k}^{\dagger}(0)\right\rangle e^{g\left(\omega_{k}\right) L} \\
& \cong \sum_{k} \frac{2 \pi \hbar \omega_{k}}{S \ell}\left[g\left(\omega_{k}\right) L+1\right]
\end{aligned}
$$

which follows from (26) and (28) and the approximation $g L<<1$ upon which (65) is based. The contribution from the term that does not vanish as $L \rightarrow 0$ can be ignored, as it corresponds to vacuum quantum noise (energy $\frac{1}{2} \hbar \omega_{k}$ per mode) that is present even in the absence of the amplifier. In other words, the quantum noise of the field in the presence of the amplifier is

$$
\begin{array}{r}
\left\langle\hat{F}\left(0, t-z / v_{g}\right) \hat{F}^{\dagger}\left(0, t-z / v_{g}\right)\right\rangle_{n} \equiv \sum_{k} \frac{2 \pi \hbar \omega_{k}}{S \ell} g\left(\omega_{k}\right) L \rightarrow \frac{\ell}{2 \pi c} \int d \omega \frac{2 \pi \hbar \omega}{S \ell} g(\omega) L \\
\cong \pi\left(\frac{2 \omega_{o} d}{c}\right)^{2} \frac{N L}{c} \int_{0}^{\infty} d \omega \frac{\beta}{\Delta^{2}+\beta^{2}}
\end{array}
$$

where we have gone to the mode continuum limit, approximated $\omega$ by $\omega_{o}$ in the numerator of the integrand, and used equation (53) for the gain coefficient. Performing the integration, we obtain exactly the noise term appearing in the denominator in the last line of (62). But now the noise is attributable to the amplification of vacuum field fluctuations [13].

Thus we can attribute the quantum noise that limits the observation of superluminal group velocity to either the quantum fluctuations of the field in the inverted medium, as do ARS, or to the quantum fluctuations of the inverted atoms, as in our derivation of the signal-to-noise ratio. The situation here is similar to that in the theory of the initiation of SF, as discussed by Polder et al. [9], or, as noted by those authors, to the theory of spontaneous emission by a single atom [14]. 


\section{Limit of Very Small Transition Frequency}

Since the origin of noise in the optical amplifier is associated ultimately with spontaneous emission, the question arises as to whether the signal-to-noise ratio might be increased by employing a transition having a very small transition frequency

$\omega_{o}$ and therefore a very large radiative lifetime. Indeed, since $\omega_{p}^{2} \propto \omega_{o}$, the second line of equation (65) suggests at first glance that $S N R \rightarrow \infty$ in the limit $\omega_{o} \rightarrow 0$. However, equation (16) shows that $v_{g} \rightarrow c$ in this limit: the superluminal effect itself becomes weaker as the spontaneous emission rate is made smaller.

In this connection we invoke once again the form (66) of the signal-to-noise ratio. If we assume $|\Delta| \tau_{p}>1$ in order that the pulse does not undergo substantial distortion as a consequence of strong absorption, then

$$
S N R(L, t)<q \frac{c \tau_{p}}{\left(v_{g}-c\right) L / c} .
$$

In other words, the signal-to-noise ratio must be smaller than the number of photons in the incident pulse times a factor equal to to length of the vacuumpropagated pulse divided by the separation of the vacuum-propagated pulse and the pulse emerging from the amplifier, independent of the the atomic transition frequency or the radiative lifetime. At the one- or few-photon level the signalto-noise ratio must therefore be less than unity under the ARS criteria for the observation of superluminal group velocity, regardless of the frequency or strength of the amplifying transition.

\section{Unitarity and Superluminal Propagation}

We now turn our attention from the specific example of the optical amplifier to some general features of superluminal propagation that follow generally from the unitary evolution of the state vector, considered here within first quantization.

The time evolution of a wave packet can be formulated in terms of a unitary operator $U(t)$ or equivalently in terms of a coordinate-space propagator $G(x-$ $\left.x^{\prime}, t\right)=\left\langle x|U(t)| x^{\prime}\right\rangle$ :

$$
\begin{aligned}
|\Psi(t)\rangle & =U(t)|\Psi(0)\rangle \\
\Psi(x, t) & =\langle x \mid \Psi(t)\rangle=\int_{-\infty}^{\infty} d x^{\prime} G\left(x-x^{\prime}, t\right) \Psi\left(x^{\prime}, 0\right) .
\end{aligned}
$$

The assumption that the propagator vanishes identically outside the light cone implies that

$$
G\left(x-x^{\prime}>c t, t\right)=0 .
$$

Given an initial wave packet centered around $x=X_{0}<0$ at $t=0$, we assume that at a later time $t>0$ it will be centered around $X_{0}+v_{g} t$, as in the example of pulse propagation in an inverted medium. 
We divide the wave packet into two parts, which we label as "superluminal" $(\mathrm{S})$ and "luminal" (L), in the following way:

$$
\Psi(x, T) \equiv \begin{cases}\Psi^{S}(x, T) & x>c T \\ \Psi^{L}(x, T) & x<c T .\end{cases}
$$

$\Psi^{S}$ vanishes if the group velocity $v_{g}<c$.

Suppose that $v_{g}>c$ and that we let the wave packet propagate for a time $T$ long enough that a superluminal signal can be clearly identified. That is, we assume that at $t=T$,

$$
\Psi^{L}(x, T) \approx 0
$$

Now

$$
\langle\Psi(T) \mid \Psi(T)\rangle=\langle\Psi(0) \mid \Psi(0)\rangle
$$

due to unitarity, and thus

$$
\int_{-\infty}^{\infty} d x^{\prime}\left|\Psi\left(x^{\prime}, 0\right)\right|^{2}=\int_{-\infty}^{\infty} d x^{\prime}\left|\Psi\left(x^{\prime}, T\right)\right|^{2} \approx \int_{c T}^{\infty} d x^{\prime}\left|\Psi^{S}\left(x^{\prime}, T\right)\right|^{2}
$$

Physically, this means that the superluminal signal, $\Psi^{S}(x, T)$, is about as large, or contains about "as many photons," as the initial wave packet.

We now combine the two underlying premises of causality and superluminal propagation as they are defined by equations (74) and (76). Using equation (73) for $x>c T$, we write

$$
\Psi^{S}(x, T)=\int_{-\infty}^{0} d x^{\prime} G\left(x-x^{\prime}, T\right) \Psi\left(x^{\prime}, 0\right)+\int_{0}^{\infty} d x^{\prime} G\left(x-x^{\prime}, T\right) \Psi\left(x^{\prime}, 0\right) .
$$

The first term vanishes because, according to Eq. (74), the integrand differs from zero only if $x^{\prime}>x-c T>0$. Thus

$$
\Psi^{S}(x, T)=\int_{-\infty}^{\infty} d x^{\prime} G\left(x-x^{\prime}, T\right)\left[\Theta\left(x^{\prime}\right) \Psi\left(x^{\prime}, 0\right)\right]
$$

This formulates the notion, which is essential to the ARS argument, that for a causal, [i.e., equation (74)], superluminal signal [equation (76)], the wave packet is reconstructed from its tail [equation (80)].

This rather remarkable reconstruction of the signal propagated without distortion and with superluminal group velocity is especially evident in the temporal domain 15. (See Figure 1.)

The construction (80) of the superluminal wave packet from the tail of the initial wave packet motivated ARS to define another, truncated initial wave packet:

$$
\Phi(x, 0) \equiv \Theta(x) \Psi(x, 0) .
$$

The two different initial wave functions, $\Phi(x, 0)$ and $\Psi(x, 0)$, give the same superluminal signal:

$$
\Psi(x>c T, T)=\Phi(x>c T, T)=\int_{-\infty}^{\infty} d x^{\prime} G\left(x-x^{\prime}, T\right) \Phi\left(x^{\prime}, 0\right) .
$$


Equation (82) implies what ARS call amplification: a "small" signal propagates to become a "large" signal. After all, $\Phi(x, 0)$ is "made from a small number of photons," while we have just seen that $\Psi(x>c T, T)$ has about the same number of photons as the non-truncated initial wave packet. We note that amplification in this sense is a necessary consequence of a superluminal group velocity.

One might be tempted to write (82) symbolically as

$$
|\Phi(0)\rangle \longrightarrow\left|\Psi^{S}(T)\right\rangle
$$

where $\longrightarrow$ denotes time evolution under $U(T)$. This would be incorrect: the truncated initial wave packet $\Phi(x, 0)$ is a perfectly well defined initial state, but it does not evolve into $\Psi^{S}(x, T)$; part of it evolves luminally. It will be prove convenient to introduce "superluminal" and "luminal" parts of the truncated wave packet in a manner similar to the decomposition (75) used for the complete wave packet $\Psi(x, T)$ :

$$
\Phi(x, T) \equiv \begin{cases}\Phi^{S}(x, T) & x>c T \\ \Phi^{L}(x, T) & x<c T .\end{cases}
$$

We note that, while the superluminal part of the time-evolved truncated initial state is the same as the superluminal part of the time-evolved non-truncated initial state, the luminal parts of these signals differ:

$$
\begin{aligned}
& \Phi^{S}(x, T)=\Psi^{S}(x, T), \\
& \Phi^{L}(x, T) \neq \Psi^{L}(x, T) \approx 0 .
\end{aligned}
$$

That is, while the luminal part of the time-evolved complete wave packet approximately vanishes $\left(\Psi^{L}(x, T) \approx 0\right)$, the luminal part of the truncated wavepacket, $\Phi^{L}(x, T)$, does not. We show below that, on the contrary, it grows exponentially with time.

\section{Momentum space: normal and unstable modes}

We are comparing the time evolution of two different initial wavepackets, $\Psi(x, 0)$ and $\Phi(x, 0)$ where $\Phi(x, 0)=\Theta(x) \Psi(x, 0)$. It is useful to define still another initial wave packet,

$$
R(x, 0)=\Theta(-x) \Psi(x, 0) \text {. }
$$

Clearly,

$$
\Psi(x, 0)=R(x, 0)+\Phi(x, 0) .
$$

After a time $T, \Psi(x, 0)$ evolves into $\Psi(x, T), \Phi(x, 0)$ into $\Phi(x, T)$, and $R(x, 0)$ into $R(x, T)$. The time evolution is linear and

$$
\Psi(x, T)=R(x, T)+\Phi(x, T) .
$$


Fourier transforming into momentum space, we define $g(k), \zeta(k)$, and $\xi(k)$, by:

$$
\begin{aligned}
& \Psi(x, t) \equiv \int_{-\infty}^{\infty} d k g(k) \exp \left[i\left(k x-\omega_{k} t\right)\right], \\
& \Phi(x, t) \equiv \int_{-\infty}^{\infty} d k \zeta(k) \exp \left[i\left(k x-\omega_{k} t\right)\right], \\
& R(x, t) \equiv \int_{-\infty}^{\infty} d k \xi(k) \exp \left[i\left(k x-\omega_{k} t\right)\right] .
\end{aligned}
$$

¿From these definitions it is straightforward to show that

$$
\begin{aligned}
& \zeta(k)=\frac{-i}{2 \pi} \int_{-\infty}^{\infty} d k^{\prime} \frac{g\left(k^{\prime}\right)}{k-k^{\prime}-i \eta}, \\
& \xi(k)=\frac{+i}{2 \pi} \int_{-\infty}^{\infty} d k^{\prime} \frac{g\left(k^{\prime}\right)}{k-k^{\prime}+i \eta},
\end{aligned}
$$

where $\eta$ is an infinitesimal positive number. ¿From the identity

$$
\frac{-1}{k-k^{\prime}-i \eta}+\frac{1}{k-k^{\prime}+i \eta}=-2 \pi i \delta\left(k-k^{\prime}\right)
$$

it follows that

$$
g(k)=\zeta(k)+\xi(k) .
$$

Equations (90)-(94) can be written as well in the following way:

$$
\begin{aligned}
\Psi(x, t) & \equiv \int_{-\infty}^{\infty} d k g(k) \psi_{k}(x, t), \\
\Phi(x, t) & \equiv \int_{-\infty}^{\infty} d k g(k) \phi_{k}(x, t), \\
R(x, t) & \equiv \int_{-\infty}^{\infty} d k g(k) \rho_{k}(x, t),
\end{aligned}
$$

where

$$
\begin{aligned}
\psi_{k}(x, t) & =\exp \left[i\left(k x-\omega_{k} t\right)\right], \\
\phi_{k}(x, t) & =\frac{-i}{2 \pi} \int_{-\infty}^{\infty} d \kappa \frac{\exp \left[i\left(\kappa x-\omega_{\kappa} t\right)\right]}{\kappa-k-i \eta}, \\
\rho_{k}(x, t) & =\frac{+i}{2 \pi} \int_{-\infty}^{\infty} d \kappa \frac{\exp \left[i\left(\kappa x-\omega_{\kappa} t\right)\right]}{\kappa-k+i \eta} \\
\psi_{k}(x, t) & =\phi_{k}(x, t)+\rho_{k}(x, t)
\end{aligned}
$$

For $t=0$ we obtain, as required by their definitions,

$$
\begin{aligned}
& \phi_{k}(x, 0)=\Theta(x) \psi_{k}(x, 0), \\
& \rho_{k}(x, 0)=\Theta(-x) \psi_{k}(x, 0) .
\end{aligned}
$$


We now invoke the premises of causality and superluminal propagation, focusing on the ARS model involving the dispersion relation

$$
\omega_{k}=c \sqrt{k^{2}-m^{2}}
$$

As long as $|k|>m$ this dispersion relation describes normal oscillating modes. Unstable modes exist for $|k|<m$. One might attempt to avoid the unstable modes altogether by choosing a $g(k)$ that vanishes or is negligibly small for $|k|<m$. This can be done, for example, by choosing an initial state with a Gaussian $g(k)$, centered around $k_{o}$ and having a width $\Delta k_{o}$ such that $\left|k_{o} \pm \Delta k_{o}\right|>>m$. This corresponds in the case of the optical amplifier to a pulse detuning large compared with a radiative decay rate. It turns out, however, as might be expected from the example of the optical amplifier, that even for such an initial wave packet $\Psi(x, 0)$ the unstable modes play an essential role in the time evolution of both the truncated and the residual wavepackets, $\Phi(x, t)$ and $R(x, t)$, respectively.

Consider the integrals in (101) and (102) as contour integrals in the complex $\kappa$ plane. The integrands, analytically continued into the complex $\kappa$ plane, each have a single, simple pole above or below the real $\kappa$ axis at $\kappa=k \pm i \eta$, and both have two branch points at $\kappa= \pm m$, which we connect with a branch cut on the line segment $(-m, m)$ on the real $\kappa$ axis. The contour from $-\infty$ to $\infty$ should pass, as usual, slightly above the real $\kappa$ axis (at a distance smaller than $\eta$ ). As shown below, this ensures causality according to equation (74). In the limit of infinite $|\kappa|$

$$
\lim _{|\kappa| \rightarrow \infty} \omega_{\kappa}=c \kappa
$$

and on the circle at infinity,

$$
\kappa x-\omega_{\kappa} t \longrightarrow \kappa(x-c t) .
$$

For $x>c t$ we can therefore close the contour integral in the upper-half plane, whereas for $x<c t$ we close the contour in the lower half. In both cases the contributions to the integral from the arcs at infinity vanish.

Using first the residue theorem for $x>c t$, we see immediately that the superluminal parts of the time-evolved residual and truncated wave packets satisfy

$$
\begin{aligned}
\rho_{k}(x>c t, t) & =0, \\
\phi_{k}(x>c t, t) & =\exp \left[i\left(k x-\omega_{k} t\right)\right] .
\end{aligned}
$$

These results are not surprising, as they simply reformulate equations (74) and (82), respectively. For $x<c t$, where we close the contour in the lower half-plane, the integral encircles the branch cut on $(-m, m)$. After deforming the contour and isolating contributions from this branch cut, we use the residue theorem and obtain

$$
\begin{aligned}
& \rho_{k}(x<c t, t)=\exp \left[i\left(k x-\omega_{k} t\right)\right]-I_{k}^{\rho}(x, t), \\
& \phi_{k}(x<c t, t)=I_{k}^{\phi}(x, t),
\end{aligned}
$$


where

$$
\begin{aligned}
& I_{k}^{\rho}(x, t)=\frac{i}{2 \pi} \int_{C} d \kappa \frac{\exp \left[i\left(\kappa x-c t \sqrt{\kappa^{2}-m^{2}}\right)\right]}{\kappa-k-i \eta} \\
& I_{k}^{\phi}(x, t)=\frac{i}{2 \pi} \int_{C} d \kappa \frac{\exp \left[i\left(\kappa x-c t \sqrt{\kappa^{2}-m^{2}}\right)\right]}{\kappa-k+i \eta}
\end{aligned}
$$

and $\int_{C} d \kappa$ is a closed contour circling counter-clockwise the branch cut on the line segment $(-m, m)$ while not circling the poles at $k \pm i \eta$. Each of the integrals, $I_{k}^{\rho}(x, t)$ and $I_{k}^{\phi}(x, t)$, is dominated by a saddle point on the imaginary $\kappa$ axis in the complex $\kappa$ plane and exponentially grows with time. Combining terms, we obtain

$$
\begin{gathered}
R(x, t)=\Theta(c t-x) \Psi(x, t)-\Theta(c t-x) \int_{-\infty}^{\infty} d k g(k) I_{k}^{\rho}(x, t), \\
\Phi(x, t)=\Theta(x-c t) \Psi(x, t)+\Theta(c t-x) \int_{-\infty}^{\infty} d k g(k) I_{k}^{\phi}(x, t) .
\end{gathered}
$$

The integrals give exponentially growing contributions to the luminal parts of both the truncated and residual wavepackets. Our choice of $g(k)$ enforces $|k \pm i \eta|>m$, and as a result,

$$
\int_{-\infty}^{\infty} d k g(k)\left[I_{k}^{\phi}(x, t)-I_{k}^{\rho}(x, t)\right]=0
$$

We see therefore that, when the residual and truncated wavepackets (115) and (116) are combined to form the complete wavepacket $\Psi(x, t)$ [Eq. (89)], the exponentially growing luminal parts cancel each other.

\section{Discussion and implications for quantum noise}

We are studying the time evolution of three wavepackets: the complete wavepacket $\Psi(x, t)$, the truncated wavepacket $\Phi(x, t)$, and the retarded, residual wavepacket $R(x, t)$. These three wavepackets can be decomposed in two different ways. In equations (90)-(94) they were decomposed in the usual way via a Fourier transform at the initial time $t=0$ into normal and unstable modes. The Fourier components of the truncated wavepacket $\zeta(k)$ and the retarded wavepacket $\xi(k)$ are related to the Fourier components of the complete wave packet, $g(k)$, by equations (93) and (94), respectively. If we choose to construct the complete wave packet from normal modes $g(k)$, where $|k| \gg m$, the truncated and retarded wavepackets will have a strong unstable-mode component in them. This was discussed by ARS, who pointed out that because of the unitarity of the time evolution, the unstable modes are accompanied by an enhancement of the quantum noise.

In order to identify the noise in a space-time picture we employed in equations (97)-(102) a less common decomposition. The difference between (97)-(102) and (90)-(94) lies in the order of integration. Both decompositions can be derived from

$$
\Psi(x, t) \equiv \int_{-\infty}^{\infty} d k g(k) \exp \left[i\left(k x-\omega_{k} t\right)\right]
$$




$$
\begin{aligned}
& \Phi(x, t) \equiv \frac{-i}{2 \pi} \int_{-\infty}^{\infty} d q \int_{-\infty}^{\infty} d p \frac{g(p) \exp \left[i\left(q x-\omega_{q} t\right)\right]}{q-p-i \eta} \\
& R(x, t) \equiv \frac{i}{2 \pi} \int_{-\infty}^{\infty} d q \int_{-\infty}^{\infty} d p \frac{g(p) \exp \left[i\left(q x-\omega_{q} t\right)\right]}{q-p+i \eta}
\end{aligned}
$$

Equation (97) describes a wavepacket made of a superposition of oscillating waves $\psi_{k}(x, t) \equiv \exp \left[i\left(k x-\omega_{k} t\right)\right]$, with the momentum distribution $g(k)$. In (98) and (99) each of these oscillating waves is replaced by a new wavefunction, $\phi_{k}(x, t)$ and $\rho_{k}(x, t)$, respectively. The weight function for the superposition forming the respective wavepackets remains $g(k)$, but $k$ has lost its meaning as a physical momentum. At any time, $\psi_{k}=\phi_{k}+\rho_{k}$. At $t=0, \phi_{k}$ and $\rho_{k}$ are obtained from $\psi_{k}$ by truncation. At a later time $t>0$, one can distinguish between two regions. In the superluminal region where $x>c t, \phi_{k}=\psi_{k}$ and $\rho_{k}=0$. In the luminal region where $x<c t, \phi_{k} \neq \psi_{k}$ : While $\psi_{k}$ is everywhere a periodic wavefunction oscillating in space and time, $\phi_{k}$ is in this region exponentially growing as a function of both $t$ and $x$; it is not oscillating in this region. In the same retarded region $\rho_{k}$ has a periodic oscillating component equal to $\psi_{k}$ and an exponentially growing component which exactly cancels the contribution of $\phi_{k}$ to this region.

The three wavepackets we consider are formed by superpositions of these different wavefunctions with the same weight function $g(k)$. They evolve in time in the following way. In the superluminal region $x>c t$ the oscillating wave functions $\psi_{k}=\phi_{k} \equiv \exp \left[i\left(k x-\omega_{k} t\right)\right]$, with $\omega_{k}$ given above, combine to form a wavepacket moving at the group velocity $v_{g}>c$; this is the superluminal signal. In the luminal region $x<c t$ the oscillating wave functions combine to cancel each other. This cancellation ensures the unitary time evolution of the complete wavepacket. The residual part of the complete wavepacket is essential for this cancellation to occur.

Using the language of truncated wavepackets introduced by ARS, we see that the superluminal signal is constructed completely from the time evolution of the forward tail, i.e., from the time evolution of the truncated wavepacket. This truncated wavepacket evolves with time into a combination of the superluminal signal and an additional, exponentially increasing part in the luminal region $x<c t$. As discussed below, this additional part that grows exponentially with time can be expected to be accompanied by substantial quantum noise, as ARS observed using a different decomposition of the same truncated wavepacket.

The new decomposition presented here therefore leads us to conclude that the exponentially growing noise is mostly "luminal" and will be delayed compared with the superluminal signal. This conclusion is consistent with the exponentially growing noise due to SF in the case of the optical amplifier [12]. Looking at the complete wavepacket, we observe that contributions from the time-evolved residual wavepacket will cancel in the luminal region $x<c t$ the contributions from the timeevolved truncated wavepacket. However, while the signal in this region vanishes by the cancellation of the two exponentially growing contributions, the noise does not cancel - and may be very large [16]. We note that an amplification of the signal in the superluminal region does occur, but our new decomposition indicates that this amplification is mostly a result of a rather efficient constructive interference of oscillating wavefunctions, while the luminal parts of the time-evolved truncated and retarded wavepackets appear to be controlled by the unstable modes. 
Our analysis in this section, being based on a "first-quantization" approach in which the wave packets are c-numbers, not operators, has not dealt explicity with quantum noise. However, as in the theory of the initiation of superfluorescence [9], the linearity of the model resulting from the approximation that there is no change in the atomic inversion over the time scales of interest allows a treatment of the operator fields as classical, fluctuating c-number fields 117 . Thus the shaded part of Figure 1a, the "tail" from which the superluminal signal evolves, becomes in such a treatment the truncated signal we have considered plus a fluctuating noise field. In the limit of a very weak incident signal pulse, the superluminal signal will be dominated by the noise part rather than the signal part of the tail shown in Figure 1a, and the signal-to-noise ratio will therefore be small, consistent with the ARS results as well as the results obtained in Section 4 for the model of an optical amplifier.

\section{Summary}

We have considered the effects of quantum noise on the propagation of a pulse with superluminal group velocity. In the case considered by CKK [1], where an offresonant, short pulse of duration $\tau_{p}$ propagates with superluminal group velocity $v_{g}$ in an optical amplifier, we calculated a signal-to-noise ratio $S N R$ and found that, for an incident pulse consisting of a single photon, $S N R<<1$ under the condition $\left(v_{g} / c-1\right) L>>\tau_{p}$ assumed by ARS [8] for discrimination between the pulse propagating in the amplifier and a twin pulse propagating the same distance in vacuum. This result is fully consistent with the conclusions of ARS based on general considerations and, in particular, the reconstruction of the superluminal pulse from a truncated portion of the initial wave packet. However, if we impose the weaker condition that $\left(v_{g} / c-1\right) L \gtrsim \tau_{p}$, then our conclusion is that $S N R>1$ is possible. However, in this case superluminal group velocity is observable in the arrival statistics of many photons, not per shot.

We showed that, in the case of the optical amplifier, the quantum noise is attributable to the onset of superfluorescence, and could be associated either with the quantum fluctuations of the field, along the lines of the ARS considerations, or with the quantum fluctuations of the atomic dipoles.

We then presented some general considerations based on unitarity and causality and introduced a new wave packet decomposition. In particular, we considered the "residual" wave packet in addition to the complete and truncated wave packets considered by ARS. This led to the conclusion that the noise is mostly luminal, that in the luminal region the truncated and residual signal grow exponentially but cancel each other as required by unitarity, but that the noise is not cancelled. For the case where the propagation time is large enough for the superluminal signal to be clearly distinguished from a twin pulse propagated at the vacuum speed of light, our conclusions were again consistent with ARS. 


\section{Acknowledgements}

We thank Y. Aharonov, E.L. Bolda, I.H. Deutsch, R.J. Glauber, P.G. Kwiat, B. Reznik, and A.M. Steinberg for helpful discussions or remarks during this work. This work was partially supported by the National Science Foundation through a grant for the Institute for Theoretical Atomic and Molecular Physics (ITAMP) at the Harvard-Smithsonian Center for Astrophysics.

\section{Appendix A}

The Heisenberg equations of motion for the Pauli operators follow from the Hamiltonian (25) and the commutation relations $\left[\hat{\sigma}_{x}, \hat{\sigma}_{y}\right]=2 i \hat{\sigma}_{z}$, etc. :

$$
\begin{gathered}
\dot{\hat{\sigma}}_{x j}=-\omega_{o} \hat{\sigma}_{y j}, \\
\dot{\hat{\sigma}}_{y j}=\omega_{o} \hat{\sigma}_{x j}+\frac{2 d}{\hbar} \hat{\sigma}_{z j} \hat{E}\left(z_{j}, t\right), \\
\dot{\hat{\sigma}}_{z j}=-\frac{2 d}{\hbar} \hat{\sigma}_{y j} \hat{E}\left(z_{j}, t\right),
\end{gathered}
$$

or, in the rotating-wave approximation,

$$
\begin{gathered}
\dot{\hat{\sigma}}_{j}=-i \omega_{o} \hat{\sigma}_{j}-\frac{i d}{\hbar} \hat{\sigma}_{z j} \hat{E}^{(+)}\left(z_{j}, t\right), \\
\dot{\hat{\sigma}}_{z j}=-\frac{2 i d}{\hbar}\left[\hat{E}^{(-)}\left(z_{j}, t\right) \hat{\sigma}_{j}-\hat{\sigma}_{j}^{\dagger} E^{(+)}\left(z_{j}, t\right)\right] .
\end{gathered}
$$

¿From the formal solution of the Heisenberg equation of motion for $\hat{a}_{k}(t)$ we obtain, using equation (26),

$$
\begin{aligned}
\hat{E}^{(+)}\left(z_{j}, t\right) & =\hat{E}_{o}^{(+)}\left(z_{j}, t\right)+\frac{2 \pi i d}{S \ell} \sum_{k} \omega_{k} \sum_{i=1}^{N_{T}} e^{i k\left(z_{j}-z_{i}\right)} \int_{0}^{t} d t^{\prime} \hat{\sigma}_{i}\left(t^{\prime}\right) e^{i \omega_{k}\left(t^{\prime}-t\right)} \\
& \equiv \hat{E}_{o}^{(+)}\left(z_{j}, t\right)+\hat{E}_{s}^{(+)}\left(z_{j}, t\right) .
\end{aligned}
$$

Here

$$
\hat{E}_{o}^{(+)}(z, t)=i \sum_{k}\left(\frac{2 \pi \hbar \omega_{k}}{S \ell}\right)^{1 / 2} \hat{a}_{k}(0) e^{-i \omega_{k} t} e^{i k z}
$$

is the homogeneous ("vacuum") solution of the Maxwell equation for the quantized

field, while $\hat{E}_{s}^{(+)}(z, t)$ is the "source" part. Now in the mode continuum limit $\sum_{k} \rightarrow(\ell / 2 \pi) \int d k=(\ell / 2 \pi c) \int d \omega$,

$\hat{E}_{s}^{(+)}\left(z_{j}, t\right)=\frac{2 \pi i d}{S \ell} \frac{\ell}{2 \pi c} \sum_{i=1}^{N_{T}} \int_{0}^{t} d t^{\prime} \hat{\sigma}_{i}\left(t^{\prime}\right) \int_{-\infty}^{\infty} d \omega \omega e^{i \omega\left(t^{\prime}-t+\left[z_{j}-z_{i}\right] / c\right)}$ 


$$
\begin{aligned}
& =-\frac{2 \pi d}{S c} \sum_{i=1}^{N_{T}} \int_{0}^{t} d t^{\prime} \dot{\hat{\sigma}}_{i}\left(t^{\prime}\right) \delta\left(t^{\prime}-t+\left[z_{j}-z_{i}\right] / c\right) \\
& \cong \frac{2 \pi i d \omega_{o}}{S c} \sum_{i=1}^{N_{T}} \int_{0}^{t} d t^{\prime} \hat{\sigma}_{i}\left(t^{\prime}\right) \delta\left(t^{\prime}-t+\left[z_{j}-z_{i}\right] / c\right) \\
& =\frac{i \pi d \omega_{o}}{S c} \hat{\sigma}_{j}(t)+\frac{2 \pi i d \omega_{o}}{S c} \sum_{i \neq j}^{N_{T}} \hat{\sigma}_{i}\left(t-\left[z_{j}-z_{i}\right] / c\right) \theta\left(z_{j}-z_{i}\right) \theta\left(t-\left[z_{j}-z_{i}\right] / c\right) \\
& =\frac{i \pi d \omega_{o}}{S c} \hat{\sigma}_{j}(t)+\hat{E}^{\prime(+)}\left(z_{j}, t\right) .
\end{aligned}
$$

Here $\hat{E}^{\prime(+)}\left(z_{j}, t\right)$ denotes the field, at the position $z_{j}$ of atom $j$, that is produced by all the other atoms of the medium.

We now use this result, and the operator identity $\hat{\sigma}_{z j} \hat{\sigma}_{j}(t)=-\hat{\sigma}_{j}(t)$, in equation (123). The result is

$$
\dot{\hat{\sigma}}_{j}(t)=-i \omega_{o} \hat{\sigma}_{j}(t)-\beta \hat{\sigma}_{j}(t)-\frac{i d}{\hbar} \hat{\sigma}_{z j}(t) \hat{E}^{(+)}\left(z_{j}, t\right)
$$

where

$$
\beta=\frac{\pi d^{2} \omega_{o}}{S \hbar c}
$$

and $\hat{E}^{(+)}\left(z_{j}, t\right)=\hat{E}_{o}^{(+)}\left(z_{j}, t\right)+\hat{E}^{(+)}\left(z_{j}, t\right)$.

Similarly, using the operator identity $\hat{\sigma}_{j}^{\dagger}(t) \hat{\sigma}_{j}(t)=\frac{1}{2}\left[1+\hat{\sigma}_{z j}(t)\right]$, we obtain from (122) and (127)

$$
\dot{\hat{\sigma}}_{z j}(t)=-2 \beta\left[1+\hat{\sigma}_{z j}(t)\right]-\frac{2 i d}{\hbar}\left[\hat{E}^{(-)}\left(z_{j}, t\right) \hat{\sigma}_{j}(t)-\hat{\sigma}_{j}^{\dagger}(t) \hat{E}^{(+)}\left(z_{j}, t\right)\right] .
$$

Since the expectation value $\left\langle\hat{\sigma}_{z}\right\rangle$ of the TLA inversion operator is $p_{2}-p_{1}=2 p_{2}-1$, where $p_{1}$ and $p_{2}$ are the lower- and upper-state probabilities, respectively, it follows that $2 \beta$ is the radiative (spontaneous emission) decay rate:

$$
\frac{1}{\tau_{R A D}}=2 \beta=\frac{2 \pi d^{2} \omega_{o}}{S \hbar c}
$$

This is not the more familiar Einstein $A$ coefficient for spontaneous emission, $A=4|\mathbf{d}|^{2} \omega_{o}^{3} / 3 \hbar c^{3}$, because it gives the spontaneous emission rate into modes propagating unidirectionally with a single polarization, whereas $A$ is the spontaneous emission rate into all possible field modes in free space. In fact $1 / \tau_{R A D}$ is the spontaneous emission rate implicit in much of laser theory: the coefficient $\lambda^{2} A / 8 \pi$ appearing in the standard expression for the gain coefficient $g(\nu)$, where $\lambda$ is the wavelength, is just $1 / \tau_{R A D}$ times the cross-sectional area $S$ :

$$
g(\nu)=\frac{\lambda^{2} A}{8 \pi}\left(N_{2}-N_{1}\right) \mathcal{L}(\nu)=\frac{1}{\tau_{R A D}}\left(N_{2}-N_{1}\right) S \mathcal{L}(\nu)
$$


for (nondegenerate) upper- and lower-level population densities $N_{2}$ and $N_{1}$, respectively, an atomic lineshape function $\mathcal{L}(\nu)$, and $|\mathbf{d}|^{2} / 3=d^{2}$.

Finally we use the definitions (28) and (30) of $\hat{F}$ and $\hat{s}$ to obtain

$$
\begin{gathered}
\hat{\dot{s}}_{j}(t)=-i(\Delta-i \beta) \hat{s}_{j}(t)-\frac{i d}{\hbar} \hat{\sigma}_{z j}(t) \hat{F}\left(z_{j}, t\right) \\
\dot{\hat{\sigma}}_{z j}(t)=-2 \beta\left[1+\hat{\sigma}_{z j}(t)\right]-\frac{2 i d}{\hbar}\left[\hat{F}^{\dagger}\left(z_{j}, t\right) \hat{s}_{j}(t)-\hat{s}_{j}^{\dagger}(t) \hat{F}\left(z_{j}, t\right)\right]
\end{gathered}
$$

in the rotating-wave approximation. The detuning between the TLA resonance frequency $\omega_{o}$ and the central field frequency $\omega$ is defined as $\Delta=\omega_{o}-\omega$. Replacing $\hat{s}_{j}(t)$ and $\hat{\sigma}_{z j}(t)$ by $\hat{s}\left(z_{j}, t\right)$ and $\hat{\sigma}_{z}\left(z_{j}, t\right)$, respectively, or $\hat{s}(z, t)$ and $\hat{\sigma}_{z}(z, t)$ in the continuum limit, we obtain equations (32) and (33).

\section{Appendix B}

For the initial state in which all the TLAs are in the upper state, $\left\langle\hat{s}_{i}\left(t_{o}\right)\right\rangle=0$ and $\left\langle\hat{s}_{i}^{\dagger}\left(t_{o}\right) \hat{s}_{j}\left(t_{o}\right)\right\rangle=\delta_{i j}$. Then the operator

$$
\hat{\mathcal{S}}=\sum_{i=1}^{N_{T}} \hat{s}_{i}\left(t_{o}\right)
$$

satisfies

$$
\begin{gathered}
\langle\hat{\mathcal{S}}\rangle=0, \\
\left\langle\hat{\mathcal{S}}^{\dagger} \hat{\mathcal{S}}\right\rangle=\sum_{i=1}^{N_{T}} \sum_{j=1}^{N_{T}}\left\langle\hat{s}_{i}^{\dagger}\left(t_{o}\right) \hat{s}_{j}\left(t_{o}\right)\right\rangle=N_{T} .
\end{gathered}
$$

In the continuum limit

$$
\begin{gathered}
\hat{\mathcal{S}}=\frac{N_{T}}{L} \int_{0}^{L} d z \hat{s}(z, 0) \\
\left\langle\hat{\mathcal{S}}^{\dagger} \hat{\mathcal{S}}\right\rangle=\frac{N_{T}^{2}}{L^{2}} \int_{0}^{L} d z^{\prime} \int_{0}^{L} d z^{\prime \prime}\left\langle\hat{s}^{\dagger}\left(z^{\prime}, t_{o}\right) \hat{s}\left(z^{\prime \prime}, t_{o}\right)\right\rangle,
\end{gathered}
$$

and we can satisfy (136) and (137) by taking

$$
\begin{gathered}
\left\langle\hat{s}\left(z, t_{o}\right)\right\rangle=0 \\
\left\langle\hat{s}^{\dagger}\left(z^{\prime}, t_{o}\right) \hat{s}\left(z^{\prime \prime}, t_{o}\right)\right\rangle=\frac{L}{N_{T}} \delta\left(z^{\prime}-z^{\prime \prime}\right) .
\end{gathered}
$$




\section{References}

[1] R. Y. Chiao, A. E. Kozhekin and G. Kurizki, Phys. Rev. Lett. 77, 1254 (1996)

[2] R. Y. Chiao, Phys. Rev. A48(1) R34 (1993).

[3] R. Y. Chiao "Population Inversion and Superluminality," in Amazing Light: A Volume Dedicated to Charles Hard Townes on his 80th Birthday, ed. R. Y. Chiao (Springer-Verlag, N. Y.,1996).

[4] A. M. Steinberg, P. G. Kwiat, and R. Y. Chiao, Phys. Rev. Lett. 71, 708 (1993); R. Y. Chiao, P. G. Kwiat, and A. M. Steinberg, Sci. Am. 269, 52 (1993); Quantum and Semiclassical Optics 7, 259 (1995).

[5] A. M. Steinberg and R. Y. Chiao, Phys. Rev. A51, 3525 (1995).

[6] Ch. Spielmann, R. Szipöcs, A. Stingl, and F. Krausz, Phys. Rev. Lett. 73, 2308 (1994).

[7] M. W. Mitchell and R. Y. Chiao, Phys.Lett. A 230, 133 (1997).

[8] Y. Aharonov, B. Reznik, and A. Stern, Phys. Rev. Lett. 81, 2190 (1998).

[9] R. Glauber and F. Haake, Phys. Lett. 68A, 29 (1978); D. Polder, M. F. H. Schuurmans, and Q. H. F. Vrehen, Phys. Rev. A19, 1192 (1979). A useful summary of the various time scales involved in superfluorescence is given by J. J. Maki, M. S. Malcuit, M. G. Raymer, R. W. Boyd, and P. D. Drummond, Phys. Rev. A40, 5135 (1989).

[10] D. C. Burnham and R. Y. Chiao, Phys. Rev. 188, 667 (1969).

[11] The factor $1 / \sqrt{\pi}$ in equation (65) is a consequence of our assumption of a Gaussian temporal profile for the incident pulse. For an arbitrary pulse shape $1 / \sqrt{\pi}$ would be replaced by some other factor less than unity.

[12] The expression (15) for the refractive index is the off-resonance approximation to the more general result $n(\omega)-1=\left(\omega_{p}^{2} \Delta / 4 \omega_{o}\right) /\left(\Delta^{2}+\beta^{2}\right)$, which gives $v_{g}=c$ in the resonant case assumed in equation (42). Thus the appropriate "observation time" for $\mathrm{SF}$ is $L / c$.

[13] The fact that we obtain the noise term appearing in the last line of (62) after an integration of the radiatively broadened gain profile over all frequencies is consistent with the assumption made in obtaining (62): the observation time was assumed to be small compared with the radiative decay time, implying a spectral bandwidth large compared with the gain bandwidth.

[14] P. W. Milonni, J. R. Ackerhalt, and W. A. Smith, Phys. Rev. Lett. 31, 958 (1973).

[15] G. Diener, Phys. Lett. A223, 327 (1996). 
[16] An analogy to a two-slit experiment can be made: The enhancement of the signal on the screen at places of constructive interference is consistent with unitarity only if the destructive interference at other places is considered as well. The noise in the dark regions, however, is not cancelled by any destructive interference of the signals from the two slits.

[17] A classical treatment of the noise in the propagation of a superluminal pulse in a gain medium has been given by E. L. Bolda, Phys. Rev. A54, 3514 (1996). For a treatment of superfluorescence in a continuously pumped medium, see also E. L. Bolda, R. Y. Chiao, and J. C. Garrison, Phys. Rev. A52, 3308 (1995). 


\section{Figure}

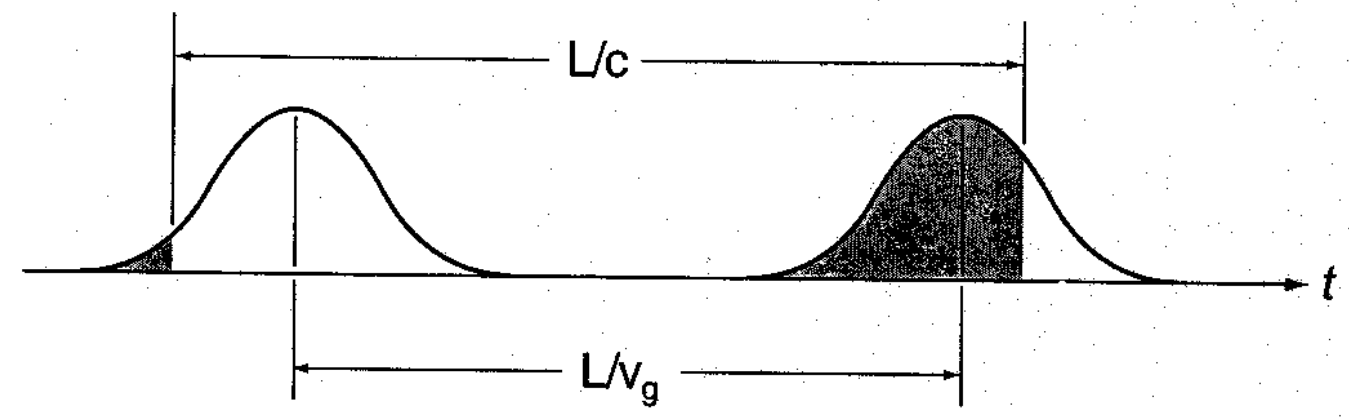

(a)

(b)

Figure 1: Incident (a) and transmitted (b) signals for a propagation length $L$ and group velocity $v_{g}>c$. It follows from the causal connection between the two signals that the shaded portion of (b) is completely determined by the shaded portion of (a). If $L\left(1 / c-1 / v_{g}\right)$ is much larger than the pulse duration, the peak of the transmitted signal is reconstructed from a small tail of the incident pulse. 